\title{
Beats Down: Using Heart Rate for Game Interaction in Mobile Settings
}

\author{
Claudia Stockhausen, Justine Smyzek, and Detlef Krömker \\ Goethe University, Robert-Mayer-Str.10, 60054 Frankfurt, Germany \\ \{stockhausen, smyzek, kroemker\} @gdv.cs.uni-frankfurt.de
}

\begin{abstract}
Mobile devices allow integration of different sensors, offering new possibilities for interaction. Integrating heart rate into a mobile game offers several possibilities for enhancing gameplay. In our work we implemented a game prototype on a mobile device with different game modes. Increasing and decreasing heart rate is used for game interaction. The mobile scenario allows involving the environment to influence the heart rate. We conducted a first user experience study for evaluation of the integrated interaction methods in mobile scenarios and conclude with our future work.
\end{abstract}

Keywords: Physiological Interaction, Mobile Games, Game Interaction.

\section{Introduction}

While physiological data has a fast growing influence in computer control, as well as in games and evaluation [2], the mobility aspect seems to be neglected. Even though mobile platforms inherit several possibilities to connect and to implement measurement sensors, the aspect of using physiological data for control and interaction within games is yet to be explored in mobile scenarios.

Many games using physiological data adapt game elements or game difficulty based on the measured data. Game mechanics can be enhanced by direct or indirect physiological control [3]. [1] describes a way to integrate different measured data into a commercial computer game engine. During gameplay different game elements and effects were adapted based on the players' physiological signals like e.g. shader effects, volume of the environment and movement speed.

An Adaptation engine, described in [9], uses physiological data for evaluation of user performance. Based on the user performance and several other parameters, like e.g. motor activity, the way of interaction can be adapted

Another field of application for physiological signals which focus more on health aspects are exertion games. In [4] heart rate is used for an exercising biathlon computer game, where the heart rate is used to control the skiing speed. "Heart Burn" [7] uses heart rate scaling to adapt game performance to the fitness level of the player. The mentioned examples are stationary and do not take advantage of interaction with physiological data in mobile scenarios. 
Only few examples exist using physiological data and taking advantage of the mobile aspect of mobile games. One example is "HeartBeat" [6], which is an outdoor pervasive game for children, in which two teams play against each other. Players wear heart rate sensors and a small device, while trying to capture members of the opposing team. Heart rate is integrated and transmitted to the small device which plays a sound, when an opponent is near and the heart rate of the opponent exceeds a given limit.

Another example is "Zone of Impulse" [5], a simple multiplayer space shooter developed for mobile devices. Galvanic skin response and heart rate are used to conclude on the user state and adapt the game difficulty to allow balancing gameplay. Both games were developed for mobile devices but does not allow to directly control the game by physiological signals or the possibility of integrating/addressing the mobile aspect/using the environment.

The game prototype, we present, uses heart rate as a means of game interaction. Gameplay can be influenced directly by increasing or decreasing the own heart rate. The novelty in our work is the aspect of taking advance of the mobility. This mobility allows the player more flexibility on how the game is played. It offers the possibility to influencing the heart rate by movements and expansive use of the environment to get a deeper gaming experience. One of the important aspects during development was to make sure the connecting devices allow a high mobility range and the minimization of user constraints and boundaries.

\section{Prototype}

The game is named "Beats Down". In order to reach a broad audience we based the game on a simple principle. The game field (figure 1) consists of 12 fields that flash randomly in red for a short time. To collect points, the gamer has to hit the flashing fields. The goal of the game is to get as many points as possible within a given time frame.

There are different ways to use the heart rate as an input for the game. Heart rate can increase, decrease or be held in a certain zone. Heart rate can even be used for further analysis of heart rate variability, which allows concluding on the mental effort of a person [8]. We decided to focus on the way a player's heart rate fluctuates, because the fluctuation can be influenced very easy by the player. Therefore, we distinguished between two game modes:

- challenge: elevate gamer heart rate to speed up game play

- relax: lower gamer heart rate to get bonus points

Furthermore one game mode without heart rate has also been integrated. In this simple mode without physiological sensing, the game only consists of hitting the flashing fields without any other input elements. 


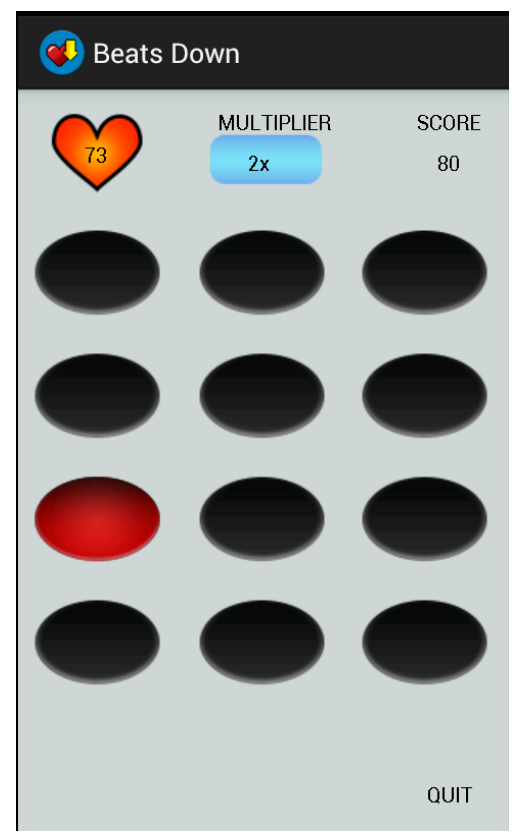

Fig. 1. Layout of Beats Down

At the beginning, the player can choose between these three game modes. If a game mode with heart rate is chosen, the heart rate is displayed at the top of the game field as an additional visual element, next to game speed or point multiplier.

\subsection{Challenge Mode}

In the challenge mode the speed of the flashing elements is controlled by the gamer's heart rate. An elevated heart rate correlates with a higher gaming speed. The elements flash up faster, but they also disappear faster. The gamer requires a faster reaction time in order to select the flashing elements, but has increased opportunities of collecting points.

The escalation of the heart rate can be achieved by physical activity or faster breathing. Due to the mobility of the game platform the player is able to engage in various physical activities to increase the heart rate.

\subsection{Relax Mode}

In the relax mode, the gamer acquires a bonus if the heart rate is decreasing. The more the heart rate is decreased in comparison to the starting heart rate, the more the point multiplier rises, resulting in a higher score for each hit. Each lowering of the heart rate by $10 \%$ in comparison to the start value is rewarded with a point multiplier bonus of 1. For example, should the heart rate be lowered by $10 \%$ in comparison to the start 


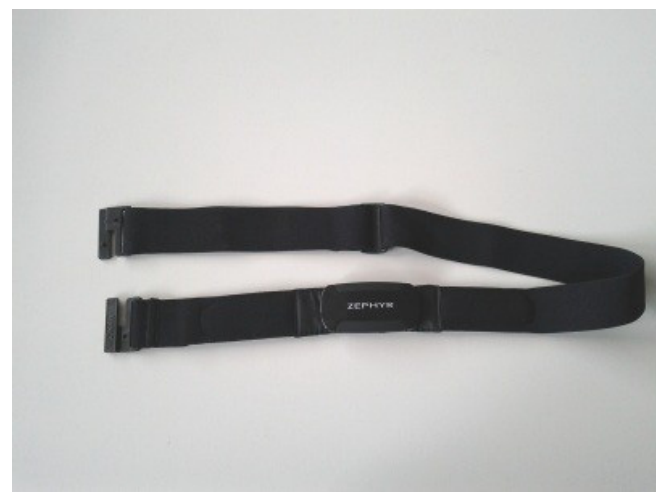

Fig. 2. Zephyr HxM heart rate monitor

heart rate, the points will double. To lower the heart rate for the relaxing mode, the gamer can take slow deep breaths or sit/lay down.

\subsection{Implementation}

The game is developed for Android 4.0.3 devices and optimized for a resolution of 470dp x 320dp. To allow mobile scenarios a sport heart rate monitor is integrated for heart rate measurement. The Zephyr HxM heart rate monitor (figure 2) transmits the data from the chest strap to the game directly via Bluetooth.

\section{Preliminary User Test}

To evaluate the game's interactivity, participants played all of the different modes of the game. The focus of the test was on the use of heart rate in the game and the aspect of mobility during gameplay. One of the goals was to monitor reactions of the participants upon using the freedom of movement for influencing the heart rate. Furthermore we wanted to find out how fun are these interactions perceived to be.

The user tests started in a usability lab, designed as a living room. Participants had enough space for complex movements and relaxing. Furthermore participants had the possibility to go outside the lab and move freely through the building or campus while playing the game modes with physiological sensing. In both situations, the participants were video recorded and all activities participants choose, were recorded.

\subsection{Experimental Procedure}

The user test is designed as a three-parameters test per test subject. The three evaluated parameters were: challenging, relaxing and a game mode without heart rate integration. The parameters were set in a randomized order. Each version of the game was played for 2 minutes and after each game session, players had a 2 minute break. 
Activities for increasing or decreasing heart rate in the different game modes were recorded.

Before the test, participants had to fill out a demographic questionnaire, answering questions about their previous gaming experience. After each session they had to fill out a questionnaire related to the current game mode and their gaming experience in that particular mode.

\subsection{Participants}

13 participants took part in the tests (11 male and 2 female). The age of the participants was between 18 and 36 with an average age of 24 . Because of health concerns, one person could not play the challenge mode. Therefore we decided to exclude this participant from the analysis of the data. On a scale from 1 to 5 ( 1 =very bad, $5=$ very good), participants rated their gaming experience on average with 3.9 and their fitness level with 3.5.

\section{$4 \quad$ Results}

Analyzing the results of the questionnaire, a Friedman test showed differences in the enjoyment ratings of the participants for the different game modes $(\chi 2(2)=16.263$, $\mathrm{p}=0.000$ ). Figure 3 shows the ratings for the different game modes concerning gratification levels experienced through the gameplay. The challenge mode was rated the highest, closely followed by the relax mode.

Investigating the ratings of game enjoyment further, a t-test between challenge and relax mode shows no significant difference $(\mathrm{p}=0.1645)$. A t-test shows a significant difference between normal mode and the challenge mode $(\mathrm{p}=0.00)$ and between normal mode and relax mode $(\mathrm{p}=0.0025)$.

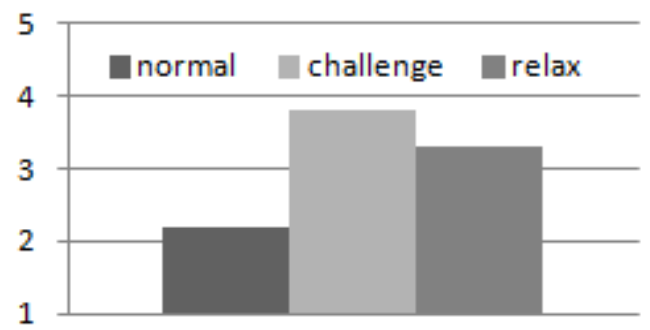

Fig. 3. Results of game enjoyment rating of the different game modes

10 of 13 participants perceived the influence of the heart rate on game play in the challenge mode. While in relax mode, 11 of 13 participants detected the influence of heart rate on their game play.

During the test, participants proved to be highly creative concerning their freedom of movement. Various activities of the participants for increasing or decreasing heart 
rate were recorded during the test (examples in figure 4). Several test subjects decided to run up and down the stairs while playing the challenge mode. Other activities in the challenge mode were: push-ups, running in the building, knee bends, running on the spot. In the relax mode one participant did yoga to raise the points multiplier. Other relaxing activities were: sitting, breathing exercises, lying down and meditation.

Questioned if they would integrate or use their environment in a mobile game, e.g. running up stairs, the mean rating was 4.07 on a five-point-scale ( $1=$ would not integrate/use it, $5=$ would integrate/use it).

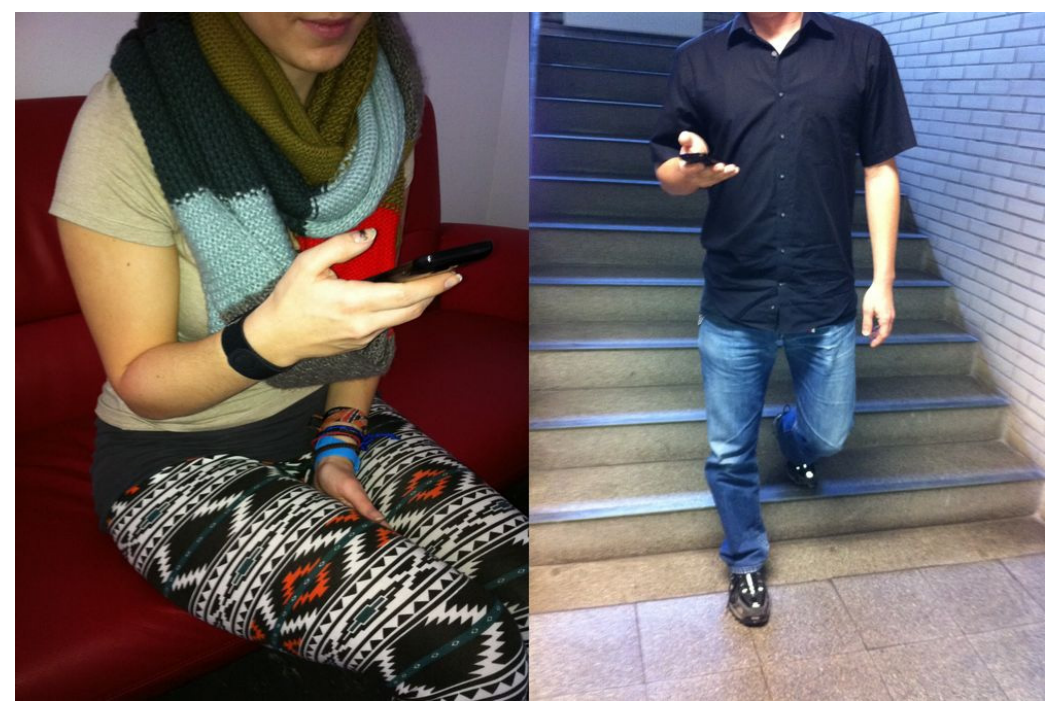

Fig. 4. Participant during relax mode (left) and during challenge mode (right)

During the user study the participants made several comments and recommendations concerning the different game modes and game usability. Most participants asked for a multi-player function, especially for the challenge mode. One participant asked to integrate different options for a bonus in the challenge mode besides game speed.

The participants were overall very interested and enthusiastic towards the implied activities and mobility aspects of the game. Furthermore they would use the offered interactions based on heart rate and enjoyment during gameplay was rated positively; specifically during the challenge mode the results showed an increase in communication and engagement.

\section{$5 \quad$ Conclusion and Future Work}

We implemented a first prototype of a mobile game, integrating different forms of interaction based on heart rate. In this game we focused only on two possible interactions but we plan to integrate several different game modes in an upcoming version of 
the game. A first short study showed that mobility can enhance physiological data integration for interaction into gameplay. Participants showed creativity regarding the use of the environment for interaction. The offered interactions based on heart rate were positively rated in the first user study.

Many possible application areas arise. For example, the mobile aspect of the integration of heart rate for interaction offers the possibility for the development of new exergames, which can be played independently from stationary devices or in combination with location based tasks. Furthermore it would be interesting to investigate further application areas, not only restricted to games.

\subsection{Future Game Modes}

After analyzing the experiment results, we would like to increase the heart rate degree of interactivity with the gameplay, giving more variation on point rewards and additional game variables. For example rewarding heart rate increases in challenge mode based on percentage with a multiplier for points, similar to the relax mode or other features in future game concepts. While in relax mode incentivize players with visual motivation even for small heart rate reductions and earn time based points for holding such achievements. Furthermore other physiological data (like electrodermal activity) can increase optional information for the challenge and relax mode. This might result in additional modes of physiological feedback and interactivity a player can choose from.

A multiplayer function can increase the challenge level between participants and give us more information about mobility and player interaction. Communication and interaction between player devices can be used for achievement, ranking or feedback purposes.

Additionally, gameplay can be combined with location based tasks, restricting the interaction based on heart rate to specific locations. For example, it can be restricted to a park or a building where you can go for a run or use the stairs.

\subsection{Future Studies}

To achieve best results and in order to find the optimal game modes, more assessments are necessary. Not only testing with single player modes but also the multiplayer options should be evaluated to verify our current findings. As an additional aspect we would like to attempt using different environments to determine player mobility limits and to expand their range of activities.

\section{References}

1. Dekker, A., Champion, E.: Please biofeed the zombies: enhancing the gameplay and display of a horror game using biofeedback. In: Situated Play Proceedings of DiGRA 2007 Conference, pp. 550-558 (2007)

2. Mandryk, R.L.: Physiological Measures for Game Evaluation. In: Isbister, K., Schaffer, N. (eds.) Game Usability. Morgan Kaufmann (2008) 
3. Nacke, L., Kalyn, M., Lough, C., Mandryk, R.L.: Biofeedback game design: using direct and indirect physiological control to enhance game interaction. In: Proceedings of the SIGCHI Conference on Human Factors in Computing Systems (CHI 2011), pp. 103-112. ACM (2011)

4. Nenonen, V., Lindblad, A., Häkkinen, V., Laitinen, T., Jouhtio, M., Hämäläinen, P.: Using heart rate to control an interactive game. In: Proceedings of the SIGCHI Conference on Human Factors in Computing Systems (CHI 2007), pp. 853-856. ACM (2007)

5. Reitz, K., Stockhausen, C., Krömker, D.: Zone of impulse: physiological data enhanced gaming. In: Proceedings of the 14th International Conference on Human-computer Interaction with Mobile Devices and Services Companion (MobileHCI 2012), pp. 221-224. ACM (2012)

6. Magielse, R., Markopoulos, P.: HeartBeat: an outdoor pervasive game for children. In: Proc. of the 27th International Conference on Human Factors in Computing Systems, pp. 21812184. ACM (2009)

7. Stach, T., Graham, T.C., Yim, J., Rhodes, R.E.: Heart rate control of exercise video games. In: Proc. of Graphics Interface 2009, pp. 125-132. Canadian Information Processing Society (2009)

8. Rowe, D.W., Sibert, J., Irwin, D.: Heart rate variability: indicator of user state as an aid to human-computer interaction. In: Karat, C.-M., Lund, A., Coutaz, J., Karat, J. (eds.) Proceedings of the SIGCHI Conference on Human Factors in Computing Systems (CHI 1998), pp. 480-487. ACM Press/Addison-Wesley Publishing Co., New York (1998)

9. Duarte, L., Carriço, L.: Coupling interaction and physiological metrics for interaction adaptation. In: Campos, P., Graham, N., Jorge, J., Nunes, N., Palanque, P., Winckler, M. (eds.) INTERACT 2011, Part IV. LNCS, vol. 6949, pp. 499-502. Springer, Heidelberg (2011) 